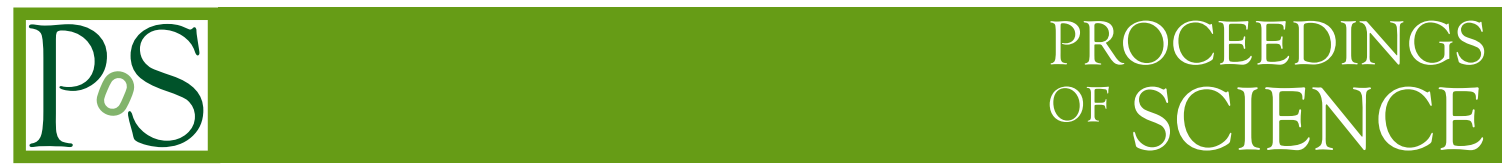

\title{
Schwinger-Dyson equations and disorder
}

\section{Adam P. Szczepaniak* ${ }^{* \dagger}$}

Physics Department and Center for Exploration of Energy and Matter, Indiana University,

Bloomington, IN 47403 USA

E-mail: aszczepa@indiana.edu

In this talk I will discuss three simple partition function models in $D=0+0$ and $D=0+1$. The goal is to compare exact results for correlation functions in these models with results of standard approximations: the saddle-point approximation and solutions of a truncated set of SchwingerDyson equations.

International Workshop on QCD Green's Functions, Confinement and Phenomenology, September 05-09, 2011

Trento Italy

\footnotetext{
${ }^{*}$ Speaker.

${ }^{\dagger}$ Supported in part by the U.S. Department of Energy under Grant No. DE-FG02-87ER40365.
} 


\section{Introduction}

There is ample evidence from lattice simulations that confinement in the QCD vacuum has origin in disorder of (chromo)magnetic domains [1, 2, 3, 4]. Condensate of magnetic charges screens electric flux lines and produces a finite gluon-gluon correlation length, i.e. magnetic mass. The gluon propagator has been extensively studied using approximate solutions to a set of truncated Schwinger-Dyson equations (SDE) [5, 6, 7]. It is therefore worth examining to what extent topological features are manifested in the Green's functions. Examples of such studies in the context of the gluon propagator, can be found in $[8,9,10,11]$.

In this talk we summarize results of studies of simple models of non-trivial vacuum, where SDE solutions can be compared to exact results. The goal is to investigate whether a set of truncated SDE can adequately capture the underlying, nontrivial properties of the vacuum. In more realistic models with nontrivial topology i.e. the Schwinger model $[12,13]$ or the abelian Higgs model [14, $15,16,17]$ Green's functions have been studied using semiclassical approximations by introducing dual variables that account for the topological defects. Here instead we will use models in which we can compare Schwinger-Dyson, semiclassical and exact results.

\section{2. $D=0+0$, one global minimum}

We begin with the case where it is expected that correlation functions will be well approximated by a truncated SDE's. That is the case when the action has one global minimum and it can be approximated by functional integral is dominated by a single and the functional integral can be then approximated by a Dyson series. In particular we consider a $D=0+0$ dimension generating functional which is a function of a single source variable, $j$ and given by a

$$
Z(j)=e^{W(j)}=\int d x e^{-S(x)+j x}
$$

For the action $S(x)$ we take

$$
S(x)=\varepsilon \frac{x^{2}}{2 !}+\lambda \frac{x^{4}}{4 !}
$$

For $\varepsilon=+1$ the action has one global minimum at $x=0$, which in higher dimensions corresponds to a unique vacuum. For this reason, in the following we refer to saddle points as vacuum contributions, which can be unique, for $\varepsilon=+1$ or multiple as in the case of $\varepsilon=-1$. For $\varepsilon=-1$ there are two degenerate minima at $x= \pm \sqrt{6 / \lambda}$ with the $x=0$ point corresponding to a local maximum and we discuss this case in the following section. We have assumed that the integral over $x$ is not restricted i.e. $x$ runs over the interval $(-\infty,+\infty)$. The semiclassical approximation will be valid in the limit of small coupling $\lambda \rightarrow 0$, as can be easily seen once $x$ is rescaled via, $x \rightarrow \bar{x}=\lambda^{1 / 2} x$,

$$
S(x)=\frac{1}{\lambda}\left(\varepsilon \frac{\bar{x}^{2}}{2}+\frac{\bar{x}^{4}}{4 !}\right),
$$

and in the small- $\lambda$ limit the integral should be well approximated by the contributions from the saddle points. For $\varepsilon=+1$ the saddle point approximation is equivalent to the leading order standard, perturbative expansion in powers of $\lambda$. In particular, the two point correlation, which we define by

$$
\left\langle x^{2}\right\rangle=\frac{d^{2} \log Z(j)_{j=0}}{d j^{2}}
$$


can be easily computed by expanding Eq. (2.1) in powers of $\lambda$ with the result

$$
\left\langle x^{2}\right\rangle=1-\frac{1}{2} \lambda+\frac{2}{3} \lambda^{2}-\frac{11}{8} \lambda^{3}+\frac{34}{9} \lambda^{4}-\frac{619}{48} \lambda^{5}+O\left(\lambda^{6}\right) .
$$

For larger values of the coupling any reasonable approximation must, at least partially, re-sum the perturbative series to all orders. Since at any order of truncation in the number of effective interactions, Schwinger-Dyson equations do sum up an infinite number of insertions of $\lambda$ one expects that a solution of a truncated set of SDE's will be a better approximation compared to the truncated perturbative expansion of Eq. (2.5). The SD equations follow from the identity

$$
S^{\prime}\left[-\frac{1}{\Gamma^{(2)}(y)} \frac{d}{d y}+y\right]=-\Gamma^{(1)}(y)
$$

where $\Gamma(y)$ is the effective action defined by

$$
\Gamma(y)=W(j)-j y, \text { with } y=\frac{d W(j)}{d j} .
$$

With $W(j)$ given by Eq. (2.2), Eq. (2.6) leads to the master equation,

$$
\varepsilon y-\frac{\lambda}{6}\left(\frac{\Gamma^{(3)}(y)}{\left[\Gamma^{(2)}(y)\right]^{3}}+\frac{3 y}{\Gamma^{(2)}(y)}-y^{3}\right)=-\Gamma^{(1)}(y)
$$

from which expectation values of any function of $x$, can be generated by taking appropriate number of derivatives. In particular the SDE for the two-point correlation,

$$
\left\langle x^{2}\right\rangle=\frac{d^{2} W(j)_{j=0}}{d j^{2}}=-\frac{1}{\Gamma_{0}^{(2)}}
$$

is obtained by taking the first derivative of Eq. (2.8) and setting the source term to zero. This gives

$$
\varepsilon+\frac{\lambda}{6}\left(\left\langle x^{2}\right\rangle^{3} \Gamma_{0}^{(4)}+3\left\langle x^{2}\right\rangle\right)=\frac{1}{\left\langle x^{2}\right\rangle} .
$$

where $\Gamma_{0}^{(4)}$ is the "dressed vertex", and together with higher order vertices, $\Gamma_{0}^{(n)}=d^{n} \Gamma(y)_{y=0} / d y^{n}$ are obtained by taking more derivatives of the master equation, e.g.

$$
-\frac{\Gamma_{0}^{(4)}}{\lambda}-1=+\frac{3}{2}\left\langle x^{2}\right\rangle^{4}\left(\Gamma_{0}^{(4)}\right)^{2}+\frac{1}{6}\left\langle x^{2}\right\rangle^{3} \Gamma_{0}^{(6)}+\frac{3}{2}\left\langle x^{2}\right\rangle^{2} \Gamma_{0}^{(4)} .
$$

Most truncation schemes in applications of SDE's are based on neglecting all but a lowest few vertices. The lowest order (LO) approximation is obtained by setting $\Gamma_{0}^{(4)}=-\lambda$ i.e. to the bare vertex, and neglecting all higher order vertices. To this order

$$
\left\langle x^{2}\right\rangle=\frac{1}{\varepsilon+\frac{\lambda}{2}\left\langle x^{2}\right\rangle-\frac{\lambda^{2}}{6}\left\langle x^{2}\right\rangle^{3}} .
$$

In the next to leading order (NLO) one would keep loop dressing of the bare vertex, appearing on the rhs of Eq. (2.11) as terms containing $\Gamma_{0}^{(4)}$, while continuing to neglect vertices generated by 


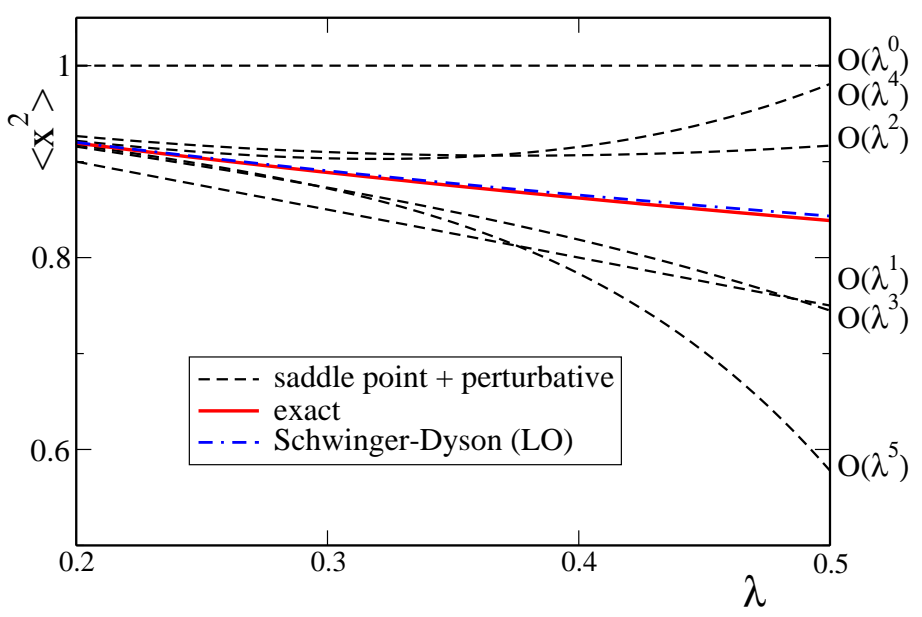

Figure 1: Comparison of perturbative (dashed lines), Schwinger-Dyson (dashed-dotted line) and exact, numerical evaluation of the two-point correlation (solid line) for the action with a unique classical vacuum $(\varepsilon=1)$. On this scale the NLO SDE solution is indistinguishable from the exact result.

higher order loops, $\Gamma_{0}^{(n)}=0, n \geq 6$. At NLO one needs to solve a set of the two coupled non-linear, algebraic equations, Eq. (2.10) and Eq. (2.11) with $\Gamma_{0}^{(6)}=0$.

It can be easily verified that the LO solution has the following expansion in powers of the coupling constant $\lambda$

$$
\left\langle x^{2}\right\rangle=1-\frac{1}{2} \lambda+\frac{2}{3} \lambda^{2}-\frac{9}{8} \lambda^{3}+O\left(\lambda^{4}\right)
$$

i.e. it agrees with the exact result only up to the second order, while for the NLO solution one finds,

$$
\left\langle x^{2}\right\rangle=1-\frac{1}{2} \lambda+\frac{2}{3} \lambda^{2}-\frac{11}{8} \lambda^{3}+\frac{34}{9} \lambda^{4}-\frac{599}{48} \lambda^{5}+O\left(\lambda^{6}\right)
$$

which agrees with Eq. (2.5) up to the fourth order. In Fig. 1 we also compare the saddle-point approximation of Eq. (2.5) with the LO and NLO solutions. Clearly the SDE equations result in the two-point correlation that is significantly more accurate then the saddle point approximation (aka perturbation theory) for large values of $\lambda$. It is worth noting that this not because the solution of the SDE's and the perturbative series match to some high order in $\lambda$; clearly the perturbative expansion is quite inaccurate except for very small $\lambda$. The agreement between solutions of SDE's and the exact result originates from the effective all order re-summation of the perturbative series generated by the nonlinear SDE's. Indeed comparing the coefficients of the first few terms in perturbative expansion beyond the order where SED's match the exact expansion, e.g. 619/48 vs $599 / 48$ one finds the difference to be only a few percent.

\section{3. $D=0+0$, multiple minima}

For $\varepsilon=-1$, SD equations truncated at any finite order cannot reproduce the exact result. 


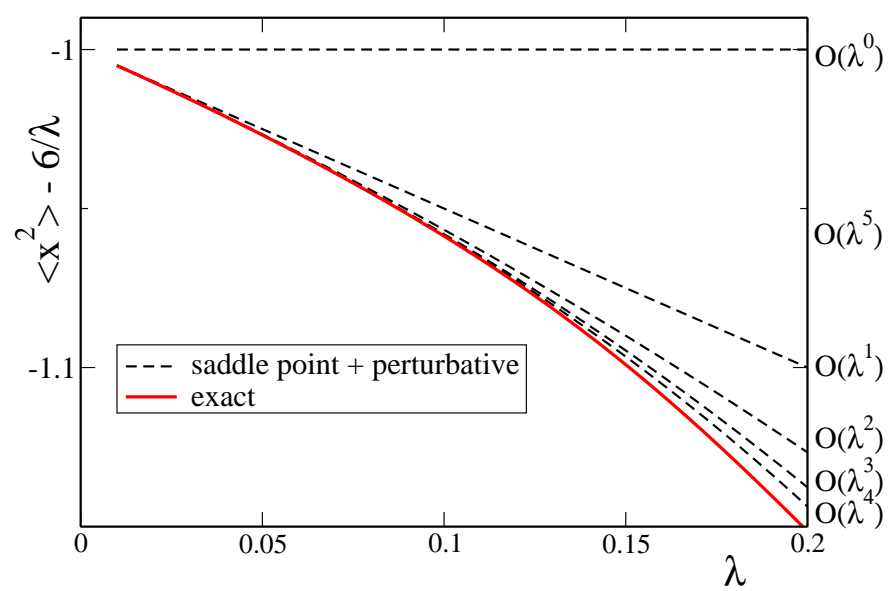

Figure 2: Saddle-point approximation computed to a varying order in $\lambda$ (dashed lines) compared with the exact result for $\varepsilon=1$ obtained by numerical integration (solid line). The $O\left(\lambda^{4}\right)$ perturbative results is indistinguishable form the exact result. For easier comparison we subtracted the leading, $6 / \lambda$ term from $\left\langle x^{2}\right\rangle$. On this scale the solution of SD equation tends to $-6 / \lambda \rightarrow-\infty$ as $\lambda \rightarrow 0$.

This is because vertices in the SDE's generated from the master equation originate from expansion around $x=0$, which is a local maximum and a metastable sate in higher dimensions. For $\varepsilon=-1$, $\left\langle x^{2}\right\rangle$ is still positive and as a function of $\lambda$ it is non-analytical at $\lambda=0$, where it has a pole, while the LO solution Eq. (2.12) is analytical and for $\lambda=0$ gives $\left\langle x^{2}\right\rangle_{L O}=-1$ ! The action has two minima and in the saddle point approximation the integral in Eq. (2.1) is approximated by a sum of gaussian fluctuations around each of them with the difference between the full action and gaussian approximation treated as perturbation. This leads to

$$
\left\langle x^{2}\right\rangle=\frac{6}{\lambda}-1-\frac{1}{2} \lambda-\frac{2}{3} \lambda^{2}-\frac{11}{8} \lambda^{3}-\frac{34}{9} \lambda^{4}-\frac{619}{48} \lambda^{5}+O\left(\lambda^{6}\right)
$$

and is compared to the exact, numerical result in Fig. 2 The analysis of the case of multiple local minima can be found in Ref. [18].

\section{Particle on a circle}

In QCD the domain of gauge fixed gluon field, i.e. the fundamental modular region may be non-flat with a non-trivial measure specified by the Fadeev-Popov determinant [19]. In the following example we investigate what happens in a model in which the dynamical variable has a non-trivial boundary under an approximation when this boundary is ignored. For this purpose we consider quantum mechanics of a free particle on a circle [20] i.e. $D=0+1$ dimensional field theory. The variable $x(t)$ now describes location of the particle on a unit circle $0 \leq x(t)<2 \pi$ and the the Hamiltonian for a particle of unit mass $H=p^{2} / 2=-\partial_{x}^{2} / 2$. Since the manifold is compact, the wave function must satisfy the boundary condition $\psi(x)=\exp (i \theta) \psi(x+2 \pi)$ and 
in the following we take $\theta=0$. The normalized eigenvectors of the Hamiltonian are spanned by $\psi_{m}(x)=\exp (\operatorname{imx} x) / \sqrt{2 \pi}$ with integer $m$ and the corresponding energies are $E_{m}=m^{2} / 2$. The vacuum expectation value at the euclidean time $(t=-i \beta)$, i.e. temperature-dependent correlation function is then given by

$$
\langle x(\beta) x(0)\rangle=\left\langle 0\left|x e^{-\beta H} x\right| 0\right\rangle=\pi^{2}+\sum_{m \neq 0} \frac{e^{-\beta \frac{m^{2}}{2}}}{m^{2}} .
$$

At low temperatures, $T=1 / \beta \rightarrow 0$ the correlation function is dominated by the lowest energy quantum sate and $\langle x(\infty) x(0)\rangle \rightarrow \pi^{2}$. In this case the restriction that $x$ be on a circle is important. At high temperatures, however, the system becomes semiclassical, and the particulars of the topology of the quantum system should become irrelevant. Also in this limit expectation values should be well approximated by contributions from small amplitude fluctuations around solutions of the classical equation of motion. In this case truncated SD equations should also be a good approximation. In our simple example we assume no interaction thus the SD equation and the semiclassical, saddle point approximation give the same results and both of them pertain to a formulation of the problem in terms of the variable dual to quantum number $m$ [21]. This variable is just is the classical coordinate $x$ and the duality transformation $m \leftrightarrow x$ is given by

$$
e^{-\beta \frac{m^{2}}{2}}=\int_{-\infty}^{\infty} \frac{d x}{\sqrt{2 \pi \beta}} e^{-\frac{x^{2}}{2 \beta}-i m x}
$$

leading to

$$
\langle x(\beta) x(0)\rangle=\int_{0}^{2 \pi} \frac{d x^{\prime}}{2 \pi} \frac{d x}{2 \pi} x^{\prime}\left\langle x^{\prime}, \beta \mid x, 0\right\rangle x
$$

where

$$
\left\langle x^{\prime}, \beta \mid x, 0\right\rangle=\sqrt{\frac{2 \pi}{\beta}} \sum_{q \in N} \exp \left(-\frac{\left(x^{\prime}-x+2 \pi q\right)^{2}}{2 \beta}\right) .
$$

At finite temperature, $q$ counts the number of times the particle wraps around the circle. The duality between $m$ and $x$ is clear; at high temperature, $\beta \rightarrow 0$ and $x$ is well defined, (while $m$ is not) since

$$
\left\langle x^{\prime}, \beta \mid x, 0\right\rangle \rightarrow 2 \pi \delta\left(x^{\prime}-x\right)
$$

with only the term with $q=0$ contributing. It then immediately follows that, $\langle x(0) x(0)\rangle=4 \pi^{2} / 3$. In the high temperature limit, the quantum variable $m$ is not well defined, i.e. in the sum in Eq. (4.1) an infinite number of terms contribute to give $4 \pi^{2} / 3$. At low temperatures, on the other hand, the system is quantum. As $\beta \rightarrow \infty, m$ becomes well defined, $m \rightarrow 0$ (while $x$ is not) and $\langle x(\infty) x(0)\rangle \rightarrow$ $\pi^{2}$. To obtain the low temperature limit of the correlation function using the classical representation of Eq. (4.4) it is necessary to integrate over the entire range of $x$ and $x^{\prime}$. Furthermore it is necessary to allow $x$ (or $x^{\prime}$ ) to wrap around the circle an arbitrary number of times i.e. sum over all $q \in N$. The approximation in which the topology of the boundary is ignored corresponds to retaining only the $q=0$ term in the sum in Eq. (4.4), which, as follows from the discussion above, should work fine in the high temperature limit but fail at low temperatures. This is shown in Fig. 3. 


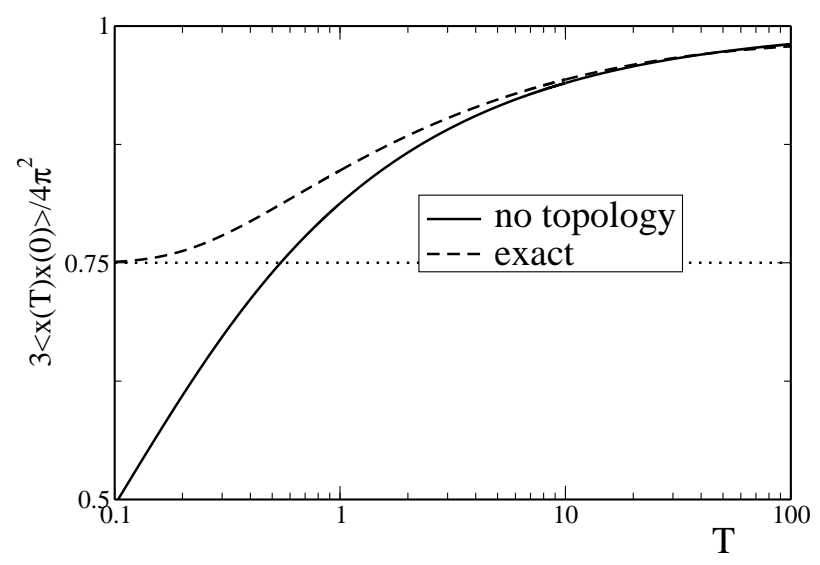

Figure 3: Comparison between exact (dashed line) and approximate evaluation of the correlation function as a function of temperature.

\section{Summary}

Since confinement is expected to be related to topologically non-trivial field configuration like magnetic monopoles or vortices it is important to access the reliability of truncation of QCD SDE's when vacuum fields are not necessarily dominated by small fluctuations around the perturbative vacuum. Here we discussed simple models to illustrate strengths and potential limitations of the SDE approach. In particular we have shown that if the partition function integral is dominated by a single semiclassical configuration, (one saddle point of the action), even the one-loop SD equation gives a much better approximation for a two-point correlation than perturbative series truncated at some high order. In the case of miltiple-saddle points or in general nontrivial topology of the ground state filed configuration, however, we expect the semiclassical approximation, may be more reliable then the truncation of the SDE series.

\section{References}

[1] Y. Nambu, Phys. Rev. D 10, 4262 (1974).

[2] S. Mandelstam, Phys. Lett. B 53, 476 (1975).

[3] A. M. Polyakov, Nucl. Phys. B 120, 429 (1977).

[4] G. 't Hooft, Nucl. Phys. B 190, 455 (1981).

[5] L. von Smekal, A. Hauck and R. Alkofer, Annals Phys. 267, 1 (1998) [Erratum-ibid. 269, 182 (1998)].

[6] C. S. Fischer, A. Maas and J. M. Pawlowski, Annals Phys. 324, 2408 (2009).

[7] A. C. Aguilar, D. Binosi and J. Papavassiliou, Phys. Rev. D 78, 025010 (2008).

[8] J. M. Cornwall, Phys. Rev. D 57, 7589 (1998).

[9] F. V. Gubarev, M. I. Polikarpov and V. I. Zakharov, Phys. Lett. B 438, 147 (1998). 
[10] M. N. Chernodub, M. I. Polikarpov and V. I. Zakharov, Phys. Lett. B 457, 147 (1999).

[11] A. P. Szczepaniak and H. H. Matevosyan, Phys. Rev. D 81, 094007 (2010).

[12] C. Adam, Czech. J. Phys. 46, 893 (1996).

[13] T. Radozycki, Phys. Rev. D 60, 105027 (1999).

[14] P. Orland, Nucl. Phys. B 428, 221 (1994).

[15] M. Sato and S. Yahikozawa, Nucl. Phys. B 436, 100 (1995).

[16] E. T. Akhmedov, M. N. Chernodub, M. I. Polikarpov and M. A. Zubkov, Phys. Rev. D 53, 2087 (1996).

[17] M. I. Polikarpov, U. J. Wiese and M. A. Zubkov, Phys. Lett. B 309, 133 (1993).

[18] A. P. Szczepaniak and H. Reinhardt, Phys. Rev. D 84, 056011 (2011) [arXiv:1106.5528 [hep-ph]].

[19] V. N. Gribov, Nucl. Phys. B 139, 1 (1978).

[20] N. Fjeldso, J, Midtdal, and F. Ravndal, J. Phys. A: Math. Gen. 21, 1633 (1988).

[21] T. Banks, R. Myerson and J. B. Kogut, Nucl. Phys. B 129, 493 (1977). 